Reprod. Nutr. Dévelop., 1980, 20 (6), 1835-1848.

\title{
Étude chronologique, structurale et biométrique de l'œuf ef de son développement chez la petite roussette (Scyliorhinus canicula) élevée en eau de mer artificielle
}

par M.M. FOULLEY, J. MELLINGER

Laboratoire de Biologie animale, Faculté des Sciences

Université de Reims, 51100 Reims, France.

Summary. The egg of the small spotted dogfish (Scyliorhinus canicula) and its development in artificial sea water : a chronologic, structural and biometric study.

The eggs of the small spotted dogfish (Scyliorhinus canicula), developed in artificial sea water at $14^{\circ} \mathrm{C}$, pre-hatched after 3 to $31 / 2$ months (total embryo length : 31 to $38 \mathrm{~mm}$ ). Hatching occurred after $61 / 2$ to $71 / 2$ months (length of newborn : 80 to $95 \mathrm{~mm}$ ). Egg weight first increased for 12 days and then remained constant until pre-hatching. A biometric study of the whole egg, egg case and albumen chamber showed very little variation in egg width, while egg length varied widely. As laying occurs in pairs, the secretory activity in each nidamental gland was observed to adapt to the extra volume of egg case contents in two cases where a double egg was laid with a normal one. However, the size and shape of the normal eggs of each female were generally distinctive, regardless of the contents or the external conditions. The egg case wall had three separate layers, as shown by microscopy of fresh material and experimental studies with various dyes.

A de rares exceptions près, les Poissons cartilagineux (Chondrichthyens = Sélaciens + Holocéphales) se reproduisent par des œufs télolécithes encapsulés (capsule ovigère, ou oothèque, ou coque) (cf. Wourms, 1977). La coque est sécrétée par une portion hautement différenciée des oviductes, la glande nidamentaire, qui fournit également son contenu muqueux (« albumen »), formé de mucopolysaccharides dont la composition rappelle la gangue des pontes d'Amphibiens plutôt que l'albumen d'un œuf d'Oiseau (Botte et al., 1976).

Comme tous les Chondrichthyens ovipares, la petite roussette (Scyliorhinus canicula) possède une coque de structure complexe, constituée de collagène associé à des scléroprotéines variées dont l'agencement et le mode de sécrétion ne sont pas encore élucidés (Knight et Hunt, 1976 ; Rusaouën ef al., 1976 ; Rusaouën, 1976, 1978). Les coques sont pondues par paires, correspondant à l'ovulation de paires d'ovocyles en règle générale. Des œufs doubles ont pourtant été signalés (Joseph, 1906 ; Vayssière, 1909). L'ovaire est unique, les deux trompes ont un pavillon commun, mais chaque glande nidamentaire reçoit l'un des ovules, dans une moitié de coque sécrétée par 
avance. Après la fécondation, l'achèvement du programme de sécrétion donne un œuf complètement fermé. L'albumen se dépose au fur et à mesure autour de cet ovule fécondé ou « jaune» (par analogie avec l'œuf d'Oiseau).

Complétant un travail antérieur (Alluchon-Gérard et Mellinger, 1971), nous précisons la chronologie du développement de cet œuf, toujours à la température standard de $14^{\circ} \mathrm{C}$, mais avec une eau de mer artificielle ef non plus naturelle comme précédemment. Dans le cadre d'une étude physiologique de la coque et de son milieu intérieur (Foulley et Mellinger, 1980), nous procédons à une comparaison à la fois biométrique et structurale entre les œufs pondus dans notre élevage expérimental par des femelles en captivité prolongée, et ceux produits à la station d'origine (Banyulssur-mer) par des femelles fraîchement capturées.

La genèse des formes très spécifiques de ces coques implique le déroulement d'un programme sécrétoire bien défini dans les glandes nidamentaires. On a noté depuis longtemps la présence occasionnelle de coques sans « jaune », élaborées en l'absence d'ovulation, de proportions normales mais de dimensions plus faibles. Ces dimensions sont-elles ajustées au diamètre des ovocytes? Comment varient-elles dans le cas d'œufs doubles? Notre étude biométrique permet de formuler certaines hypothèses sur la régulation de cette sécrétion particulièrement complexe.

\section{Matériel et méthodes.}

Dans une pièce isolée, climatisée à $14^{\circ} \mathrm{C}$ (optimum thermique d'après Wintrebert, 1920a), l'eau de mer circule dans des bacs de 1300 litres munis d'un vaste compartiment filtrant (Aquariums R. Coutant, La Rochelle). La circulation ef l'aération sont assurées par de l'air comprimé distribué à partir d'un gros compresseur à pistons, avec interposition d'un détendeur ef d'un piège à huile. Comme matériau filtrant, la laine de verre donne les meilleurs résultats. Il suffit de nettoyer les ouvertures et la surface du filtre, sans jamais changer la laine.

L'un des bacs est réservé aux opérations de renouvellement et de recyclage de l'eau des boîtes qui contiennent les cufs. La qualité de l'eau de mer est contrôlée régulièrement : densité $(d=1,030)$, absence d'odeur, de trouble, de coloration, $\mathrm{pH}$ (teinte jaune-orangé du rouge neutre), taux de nitrites (nécessaire de dosage Eheim). Malgré la parfaite stabilité des caractéristiques de l'eau, nous prenons la précaution d'un renouvellement partiel, plusieurs fois par an. Celte eau de mer artificielle est préparée d'avance à partir d'eau de ville calcaire et de sels pour l'aquariologie (Meeressalz «Wimex», H. Wiegandt, 415 Krefeld 1, République Fédérale d'Allemagne).

Les roussettes et leurs œufs sont de plus petite taille en Méditerranée que dans la Manche (Leloup et Olivereau, 1951). Nos pondeuses proviennent de la mer Méditerranée (Banyul-sur-mer), uniquement. Elles sont nourries de poisson (merlan, etc.) une fois par semaine. La mortalité est pratiquement nulle, sauf pour des roussettes ou des œufs reçus en mauvais état.

Chaque cuf est suspendu par ses vrilles antérieures (fig. 1) dans une boîte en plastique alimentaire transparent, remplie de $800 \mathrm{ml}$ d'eau de mer artificielle. La glissière du couvercle verseur est laissée ouverte pour assurer une meilleure oxygénation, mais les dosages $d^{\prime} \mathrm{O}_{2}$ ont toujours montré des valeurs proches de la saturation 
malgré l'absence d'agitation. Compte tenu de la viłesse d'apparition des nitrites, l'eau des boîtes est changée au moins une fois par semaine.

Pour la pesée, l'œuf est débarrassé de ses vrilles postérieures, les antérieures étant réduites au minimum nécessaire pour assurer la suspension dans la boîte. Il est essuyé complètement avec du papier filtre, puis rapidement pesé pour éviter toute dessiccation. La conservation de coques vides ou de fragments de coques se fait toujours en eau de mer filtrée, de préférence froide $\left(0-4^{\circ} \mathrm{C}\right)$ : on ne note aucune putréfaction ni altération quelconque, pourvu que tout le blanc ait été éliminé.

On mesure l'épaisseur des coques à l'aide d'un micromètre externe (Palmer) au $1 / 100$ de millimètre. Comme la coque résiste très mal à la déshydratation et à l'inclusion, nous y pratiquons des coupes fines à main levée, sur le frais. Les embryons sont mesurés ou fixés sous anesthésie au MS 222 (Sandoz) après l'extraction du germe hors de la coque.

\section{Résultats.}

1. Structure de l'œuf et modifications au cours du développement.

La structure générale de l'œuf de roussette et les modifications annonçant l'éclosion sont connues depuis les travaux de Wintrebert (1920a et $b, 1927 a, b$ et $c, 1931$ ) et Ouang Te Yio (1931), mais un rappel sera utile (fig. 1, 2 et 3 ).

La sécrétion des différentes parties de la coque commence par le bout des vrilles postérieures, rattachées à l'extrémité arrondie (dite « forcipéa», d'ajrès la terminologie de Wintrebert), el se termine par les vrilles intérieures, prolongeant les angles de l'extrémité quadrangulaire («spatulée »), Ces filaments vrillés permettent à la pondeuse de ligoter ses œufs à un support (en aquarium, Gux tuyaux d'aération). La forme de l'extrémité forcipée favorise la descente de l'œuf dans l'oviducte, tandis que l'extrémité opposée fournira un passage pour l'éclosion après descellement de son limbe.

L'apparence de symétrie bilatérale que présente la coque se trouve contredite par la disposition des 4 « boutonnières », fissures préformées, d'abord obturées par un albumen solide et situées le long des arêtes latérales tout près des extrémités. Dans la position conventionnelle (fig. 1), les deux boutonnières de gauche font face à l'observateur, celles de droite étant sur la face opposée.

Le «jaune» télolécithe repose dans la chambre chalazifère ménagée dans la partie postérieure de l'albumen et nommée ainsi par Wintrebert en raison de la présence de deux boyaux axiaux

FIG. 1. - Schéma d'un œuf de roussetfe, avant la digestion de l'albumen. Les vrilles ne sont pas figurées en entier. $V i$, masse vitelline; e, embryon ; ch, partie libre de la chambre chalazifère; $a$, chalaze antérieure ; $p$, chalaze postérieure ; b, boutonnières. Le même œuf est visible sur la figure 2 .

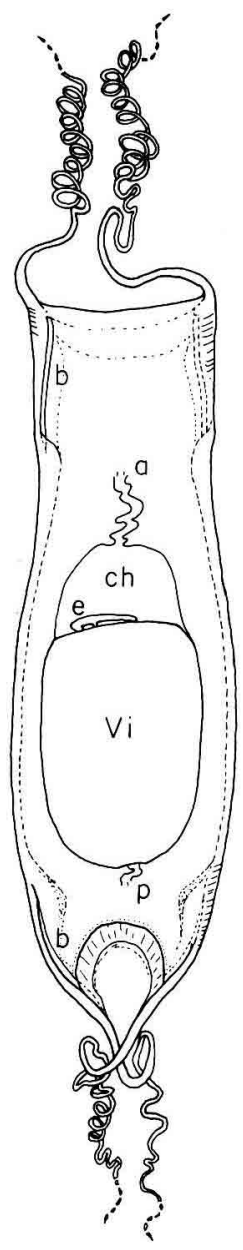


sinueux qui évoquent les chalazes de l'œuf d'Oiseau par leur rôle apparemment suspenseur. En réalité, Wintrebert a poussé la comparaison trop loin. S'il est exact que l'albumen présente une zone centrale assez fluide (" glaires » mobiles dans un liquide interstitiel) ef une couche corticale gélifiée (" gluten»), le rôle d'ancrage conféré aux chalazes demande encore à être vérifié. En tout cas, la position et les dimensions de la chambre demeurent absolument fixes même lorsqu'on retourne l'œuf. Par contre, le « jaune » s'y meut aisément de manière à s'orienter suivant le gradient vitellin. II n'occupe que les trois quarts de la hauteur de la chambre, dont il adopte partiellement les contours elliptiques (fig. 2). La forme du pôle antérieur du «jaune », d'abord arrondie, devient plus plate (fig. 2) après la rupture de la membrane vitelline, qui intervient aux stades où l'embryon mesure 3 à $5 \mathrm{~mm}$ de longueur (Wintrebert, 1927c).

Vers le 75 e jour après la ponte, on note le début de la digestion de l'albumen qui se manifeste par la disparition des parois de la chambre chalazifère. Cette profonde transformation est due aux enzymes sécrétées par la glande de l'éclosion siłuée sur le rostre de l'embryon (Ouang Te Yio, 1931). Durant les 20 à 35 jours suivants, les glaires puis le gluten sont dissous. La position de la masse vitelline par rapport à la coque change alors d'une manière très caractéristique (fig. 3 ).

Cette digestion finit par l'élimination du gluten solide qui obturait les boutonnières et scellait les lèvres de l'extrémité d'éclosion. L'eau de mer peut alors circuler librement dans la coque, facilitant les échanges d'un embryon devenu assez volumineux. Dans notre élevage, cette ouverture, facile à constater par une simple pression des doigts sur la coque, peut se produire du $93^{\mathrm{e}}$ au $112^{\mathrm{e}}$ jour après la ponte, selon les œufs, alors que l'éclosion n'a lieu qu'entre le $200^{\circ}$ et le $230^{\circ}$ jour. Cette particularité des Chondrichthyens permet de définir l'ouverture de la coque comme une " prééclosion » (Alluchon-Gérard et Mellinger, 1971), marquant la mi-temps du développement embryonnaire. C'est à partir de ce stade, ou peu de temps avant, que l'embryon supporte parfaitement l'élevage hors de la coque, et il suffit de le placer directement sur le fond du bocal avec sa vésicule vitelline déjà bien formée.

\section{PLANCHE}

FIG. 2. - Aspect d'un œuf avant la digestion de l'albumen. E, embryon ; Vi, masse vitelline.

FIG. 3. - Aspect d'un œuf après digesfion de la majeure partie de l'albumen, peu de temps avant la prééclosion. Un front de digestion du gluten est nettement visible à l'avant (triangles). La vésicule vitelline externe (VVE) repose sur le front postérieur et entre en contact avec les parois latérales de la coque. A gauche, on note la présence de vrilles rebroussées derrière la coque. E, embryon (extrémité céphalique) ; F, filaments branchiaux externes.

FIG. 4. - Coupe dans une face de la coque, montrant les trois couches de nature différente : $E$, couche externe; $M$, couche moyenne (lamelleuse) ; i, couche interne (entre les deux triangles, liseré coloré exclu). Echelle : $100 \mu \mathrm{m}$. Cette coupe a été réalisée à main levée, dans un morceau de coque frais, préalablement immergé dans le vert lumière durant $1 \mathrm{~h}$ (seule la couche externe est colorée).

FIG. 5. - Diffusion du vert lumière dans la couche moyenne, à partir d'une rayure de la couche interne de la coque (entre les deux triangles). La rayure, courbe, se poursuit jusqu'en haut de la micrographie : le colorantforme un chapelet de taches, car la rayure ne traverse pas partout la couche interne. Des images de diffusion IIre latérale anisotrope sont également visibles (vers le carré). Echelle : $100 \mu \mathrm{m}$. 


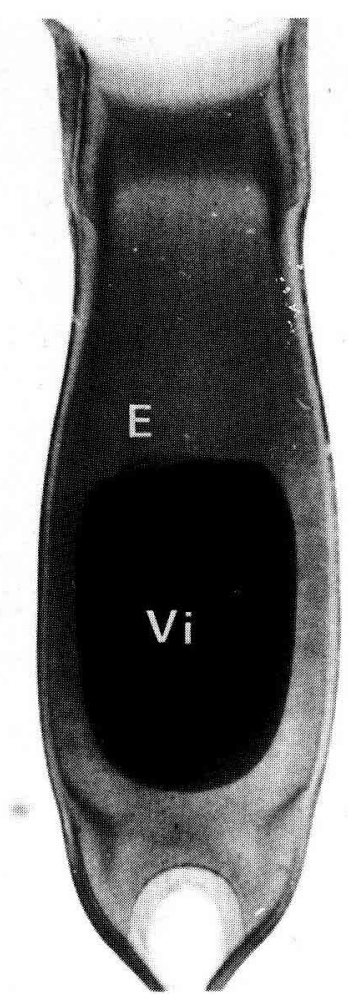

2

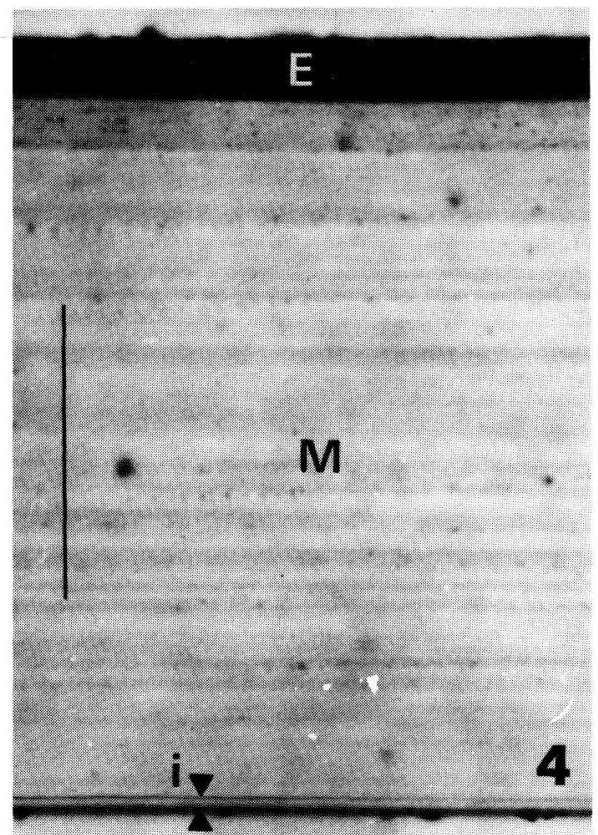

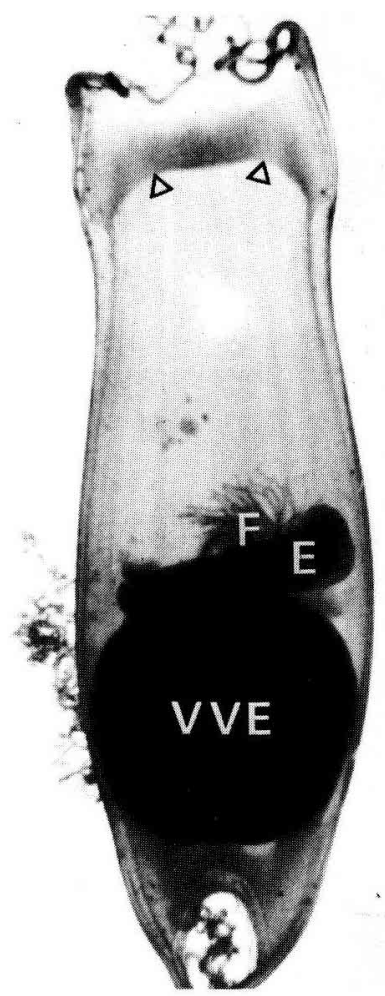

3

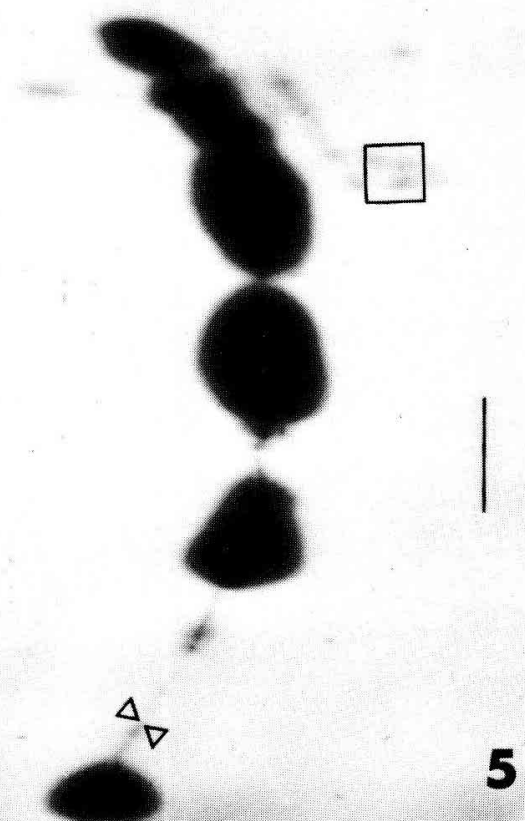


Dix embryons mesurés vivants (anesthésiés) au moment de la pré-éclosion atteignaient des longueurs totales de 31 à $38 \mathrm{~mm}$ (onze autres, fixés au Bouin-Hollande ef lavés à l'éthanol à $70^{\circ}: 25-32 \mathrm{~mm}$ ). Six nouveau-nés mesuraient 81 à $88 \mathrm{~mm}$, mais les stades morphologiques correspondants, observés chez les embryons élevés hors de leur coque (fig. 6 et 7), peuvent couvrir un intervalle de tailles allant de 80 à $95 \mathrm{~mm}$. Suivant une règle bien établie chez des Sélaciens variés, ovipares ou vivipares, la naissance intervient après la résorption de la vésicule vitelline externe (VVE). Chez la roussette, un bouton de 1-2 $\mathrm{mm}$ peut persister. Cette résorption correspond à un transfert du vitellus non consommé, à travers le pédicule vitellin, dans uñ vésicule vitelline interne. Dans notre élevage, le transfert commence vers le $150^{\mathrm{e}}$ ou le $180^{\mathrm{e}}$ jour, aux environs du stade $65 \mathrm{~mm}$. Jusque-là le diamètre de la VVE ne diminue guère (cf. fig. 6 et 7 ).

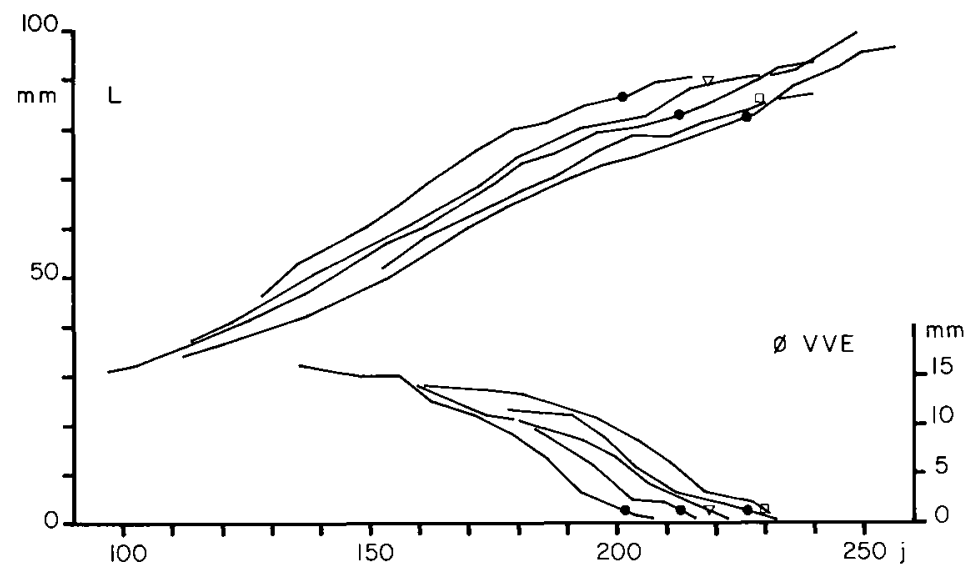

FIG. 6. - Courbes de croissance d'embryons élevés hors de leurs coques après la pré-éclosion (en haut ; $\mathrm{L}$, longueur totale en $\mathrm{mm}$; j, nombre de jours après la ponte). En bas, courbes de régression des vésicules vitellines externes ( $\varnothing \mathrm{VVE}$, diamètre de la vésicule en $\mathrm{mm}$ ). Sur les courbes, des symboles indiquent le stade morphologique correspondant à l'éclosion naturelle et permettent d'associer chacune des courbes du haut à l'une du bas de la figure. Choix de courbes extrait d'une vingtaine de courbes similaires.

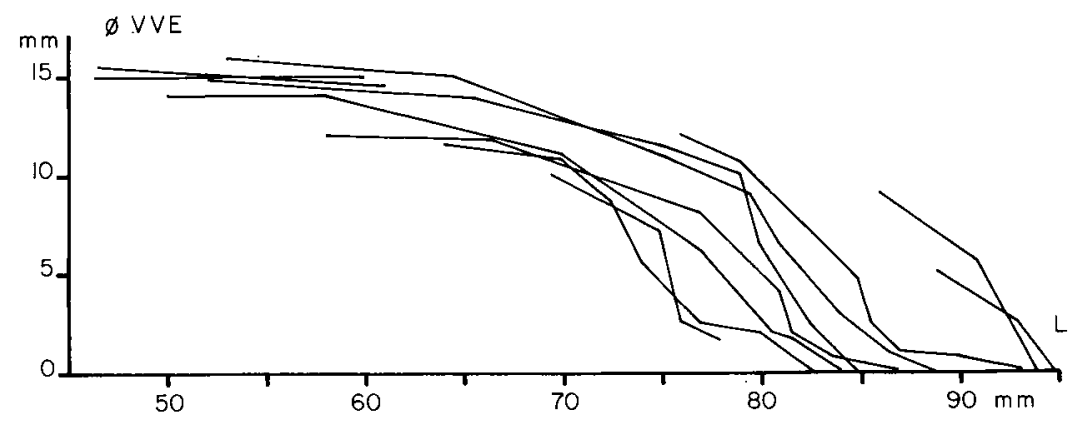

FIG. 7. - Autres courbes de régression de la VVE, où le diomètre ( $\varnothing$ VVE, en $\mathrm{mm}$ ) est représenté en fonction de la longueur totale de chaque embryon (L, en $\mathrm{mm}$ ), ce qui indique la possibilité d'une variation de tailles allant de 80 à $95 \mathrm{~mm}$ pour les nouveau-nés. 
La structure, la surface et le poids de la coque proprement dite ne changent pas sous l'effet de la digestion de l'albumen. Des détails sur le mode d'éclosion sont donnés dans une note préliminaire (Mellinger et Foulley, 1979).

\section{Couches constitutives de la coque et action des colorants.}

Dans des coupes transversales faites à main levée dans les parois de la coque, à l'état frais, on peut distinguer trois couches. Leur colorabilité par les divers colorants que nous avons employés sous la forme de solutions concentrées (environ $50 \mathrm{~g} / \mathrm{l}$ d'eau de mer) confirme bien qu'il s'agit là de trois formations nettement différentes les unes des autres (tabl.).

\section{TABLEAU}

Pénétration des colorants dons les trois couches de la coque de l'œuf

Type PM C.externe C.moyenne $\begin{gathered}C . \\ \text { interne }\end{gathered}$

\section{Colorants acides}

\begin{tabular}{|c|c|c|c|c|c|c|c|}
\hline 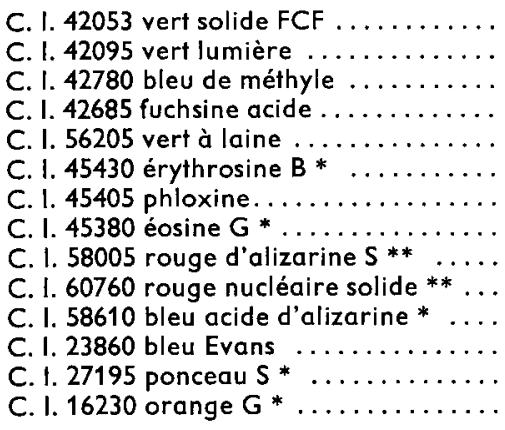 & $\begin{array}{l}\text { TPM } \\
\text { TPM } \\
\text { TPM } \\
\text { TPM } \\
\text { TPM } \\
\text { XAN } \\
\text { XAN } \\
\text { XAN } \\
\text { AQI } \\
\text { AQI } \\
\text { AQI } \\
\text { DIA } \\
\text { DIA } \\
\text { DIA }\end{array}$ & $\begin{array}{l}809 \\
793 \\
756 \\
585 \\
577 \\
880 \\
721 \\
692 \\
342 \\
335 \\
291 \\
961 \\
760 \\
452\end{array}$ & $\begin{array}{l}+++ \\
++ \\
+ \\
+ \\
++-t \\
+++ \\
+++ \\
++ \\
++\end{array}$ & $\begin{array}{l}D \\
D\end{array}$ & $\begin{array}{l}D \\
D\end{array}$ & $\begin{array}{l}+ \\
+ \\
0 \\
+++(40) \\
+++ \\
0 \\
++(1 j) \\
++(1 j) \\
+ \\
+ \\
0 \\
0 \\
+ \\
+++\left(15^{\prime}\right) D\end{array}$ & $\begin{array}{c}0 \\
\pm \\
+t \\
+ \\
\pm \\
0 \\
D+-\end{array}$ \\
\hline \multicolumn{8}{|l|}{ Colorants basiques } \\
\hline 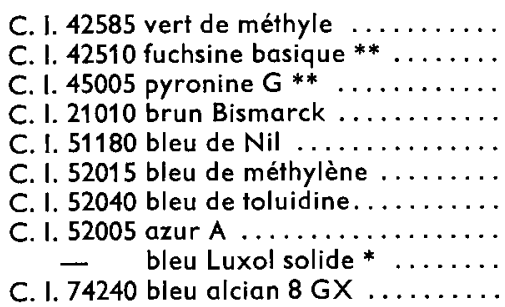 & $\begin{array}{l}\text { TPM } \\
\text { TPM } \\
\text { XAN } \\
\text { DIA } \\
\text { OXA } \\
\text { PHE } \\
\text { PHE } \\
\text { PHE } \\
\text { PHT } \\
\text { PHT }\end{array}$ & $\begin{array}{r}458 \\
324 \\
303 \\
419 \\
415 \\
320 \\
306 \\
292 \\
1000 \\
1342\end{array}$ & $\begin{array}{c}++ \\
+++ \\
++ \\
+++ \\
+++ \\
++++ \\
+++ \\
+ \\
0\end{array}$ & $\begin{array}{l}d \\
D\end{array}$ & $\begin{array}{l}d \\
D\end{array}$ & $\begin{array}{l}0 \\
\pm(1 \mathrm{j}) \\
\pm(1 \mathrm{j}) \\
\pm(1 \mathrm{j}) \\
0 \\
++(2 \mathrm{~h}) \\
++(1 \mathrm{j}) \\
+-(1 \mathrm{j}) \mathrm{d} \\
0 \\
0\end{array}$ & $\begin{array}{l}0 \\
+ \\
+ \\
++ \\
+ \\
+ \\
+ \\
++ \\
0 \\
+\end{array}$ \\
\hline
\end{tabular}

Les colorants, dont les noms sont précédés de leur numéro du « Color Index 》 (C. I.), sont classés dans l'ordre décroissant des poids moléculaires (PM) au sein de chacune des familles chimiques (types: $T P M$, dérivés du triphénylméthane ; XAN, dérivés du xanthène ; $A Q 1$, dérivés de l'anthraquinone : DIA, diazoïques; OXA, oxazines; PHE, phénothiazines ; PHT, phtalocyanines).

La pénétration dans les couches superficielles (c. externe et c. interne) a été appréciée d'après l'examen de l'intensité relative des taches obtenues sur les faces externe ou inferne d'une coque après le dépôt de gouttelettes des solutions colorantes, un séjour de 4 heures en chambre humide et un bref lavage à l'eau de mer. Echelle des intensités: $++++==$ très forte,+++ forte, $++==$ moyenne, $f==$ faible, \pm très faible, $0=$ nulle. La solubilité faible * ou même très faible ** de certains colorants $n$ 'a pas compromis le résultat. L'auréole de diffusion observée autour de certaines taches (D, diffusion forte ; $d$, légère) est généralement liée à une pénétration dans la couche moyenne, sous-jacente, ce qu'on vérifie sur des coupes (D-D, d-d). La pénétration dans la couche moyenne a également été étudiée par trempage de fragments de coques dans le colorant. Sauf mention contraire (parenthèse), le résultat est donné au bout d'une heure. 
On voit que la couche externe se colore beaucoup mieux que la couche interne. Cette dernière retient la plupart des colorants basiques et, parmi les colorants acides, on notera son affinité pour les anthraquinones. Ni la solubilité ni le poids moléculaire des colorants ne semblent déterminants pour la colorabilité. Seul l'orange $G$ pénètre dans la couche moyenne avec une égale facilité à partir de l'une ou l'autre des couches adjacentes.

L'épaisseur des faces de la coque varie de 0,22 à $0,48 \mathrm{~mm}$ en son centre, selon les œufs. Nous avons noté une différence entre ceux pondus en élevage $(0,30$ à $0,48 \mathrm{~mm})$ ef ceux en provenance de Banyuls $(0,22$ à $0,35 \mathrm{~mm})$. En coupe (fig. 4), dans une région dont l'épaisseur totale est de $260-270 \mu \mathrm{m}$, la couche externe mesure $20 \mu \mathrm{m}$, la couche interne 3-4 $\mu \mathrm{m}$, le reste (environ $245 \mu \mathrm{m}$ ) correspondant à la couche moyenne, composée de nombreuses sous-unifés. D'après Knight ef Hunt (1976), l'épaisseur d'une lamelle élémentaire serait de $0,5 \mu \mathrm{m}$ dans une coupe ultrafine perpendiculaire, étudiée au microscope électronique : on ne peut donc guère espérer les compter au microscope photonique, mais nous présumons qu'il doit en exisłer environ 500 . L'aspect de «lames», d'épaisseur décroissante de l'extérieur vers l'intérieur ef dont Fauré-Fremiet (1938) fixait le nombre à 25-30 chez la roussette, traduit sans doute l'existence d'une variation périodique dans l'orientation des protéines fibreuses. Nous ne trouvons aucune subdivision naturelle constante pour cette couche principale de la coque.

Ces trois couches sont d'ailleurs discernables sans coloration. La couche externe, blanchâtre et opalescente sans doute en raison de sa structure granuleuse (Knight et Hunt, 1976), comporte des striations longitudinales caractéristiques de cet cuf. On sait depuis longtemps que son élimination au rasoir permet de créer des fenêtres transparentes pour observer l'embryon (Wintrebert, 1920a). Ceci indique que les autres couches sont transparentes. Nous avons vérifié que la couche moyenne, cristalline, confère à l'œuf sa coloration, variant du brun foncé au jaune très clair. La couche interne semble jouer un rôle mécanique important de par sa surface interne parfaitement lisse qui ménage la fragilité de l'ovule.

La figure 5 montre l'effet d'une lésion accidentelle (rayure) de la couche interne : on observe la pénétration du vert lumière par les brèches el de là sa diffusion dans la couche moyenne. Cette diffusion a lieu d'une manière isotrope, tant que les lamelles n'onf pas subi de dissociation traumatique.

\section{Poids, dimensions, surface et épaisseur de la coque ou de l'œuf.}

Le poids de huit œufs a été contrôlé quotidiennement à partir du moment de l'oviposition et jusqu'à la pré-éclosion. Nous pensions que la digestion de l'albumen, à partir du $75^{\mathrm{e}}$ jour, pouvait modifier la pression osmotique interne et créer un appel d'eau à travers la coque. Mais la pesée démontre la stabilité parfaite du poids des œufs à partir du $12^{\mathrm{e}}$ jour et jusqu'à la pré-éclosion.

Durant les 12 premiers jours (fig. 8), on note une augmentation régulièrement amortie, dont l'importance dépend du poids initial des différents œufs : elle varie de 0,4 à 1,1 g, ce qui correspond à 10-18 p. 100 du poids final. Le poids total retenu par la suite sera toujours le poids final, déterminé avant la pré-éclosion. 


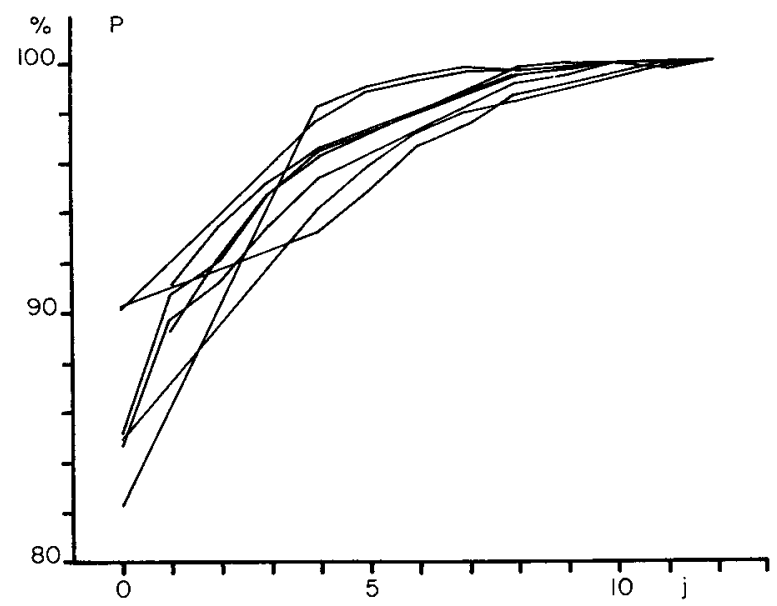

FIG. 8. - Augmentation du poids de 8 œufs (en p. 100 du poids final, $\% \mathrm{P}$ ) durant les 7 à 12 premiers jours après la ponte.

Dans les graphiques suivants (fig. 9, 10 et 11), on constate que les œufs pondus en élevage à Reims sont généralement plus grands et plus lourds que ceux recueillis à Banyuls. Ces cufs sont parfaitement viables et ne présentent aucune anomalie particulière dans leur développement.

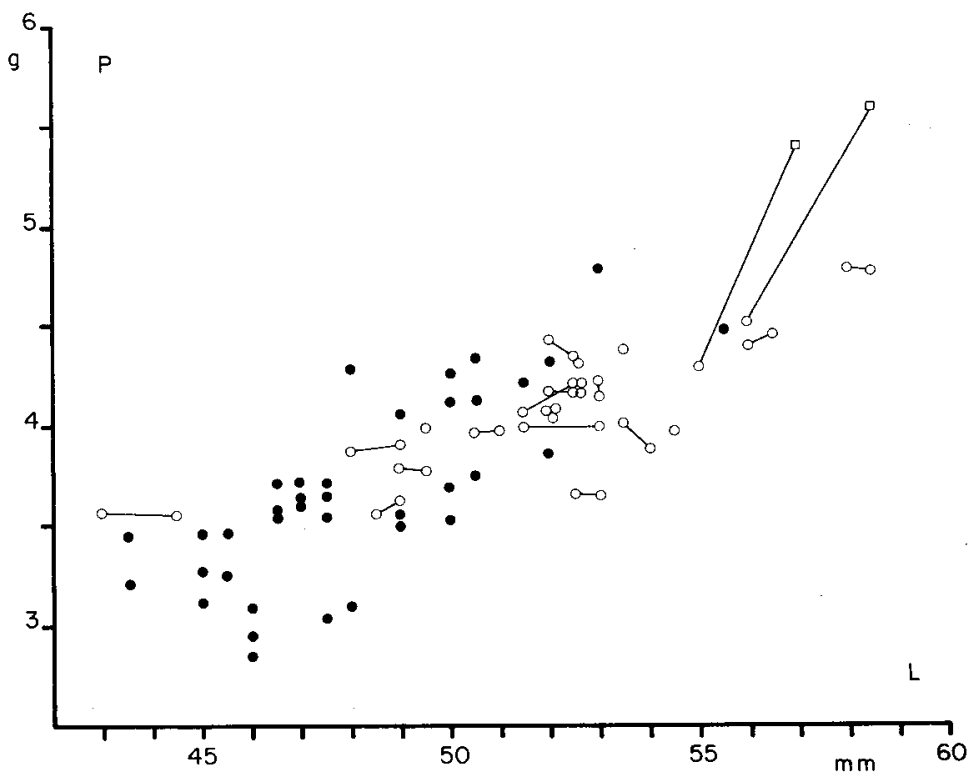

FIG. 9. - Relation poids (P, en $g$ ) ; longueur ( $L$, en $\mathrm{mm}$ ) de l'œuf. Points : œufs pondus à Banyuls ; cercles et carrés : œufs pondus à Reims (paires reliées d'un trait); carrés : deux œufs doubles. Relation très significative $(r=0,8)$. 


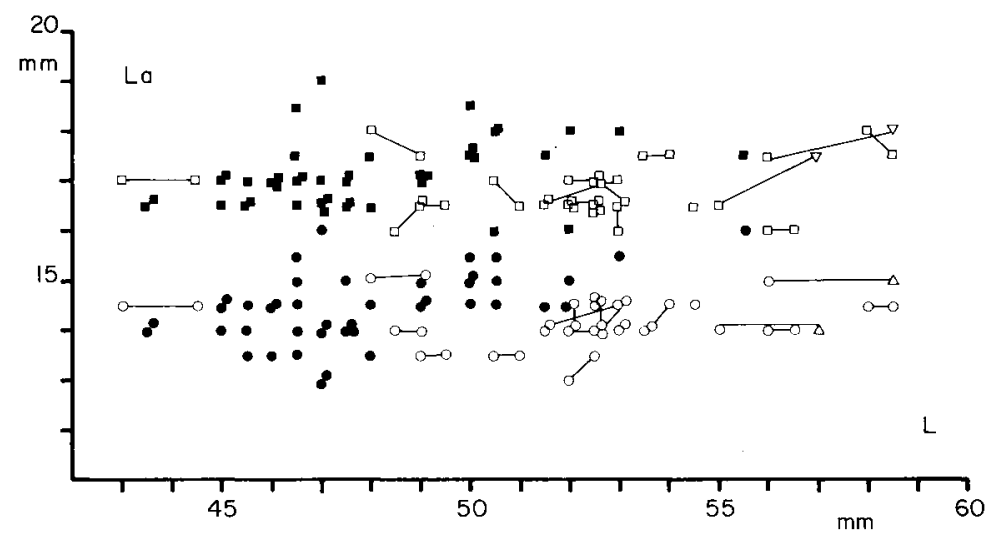

FIG. 10. - Relations largeur (La) - longueur ( $\mathrm{L}$ ) de l'œuf. Deux relations : en haut, largeur maximum mesurée dans la zone renflée de la coque (carrés noirs : œufs de Banyuls ; carrés blancs : œufs de Reims) ; en bas, largeur du bout spatulé (points noirs : Banyuls ; points blancs : Reims). Triangles : deux œufs doubles. Les œufs d'une même paire sont reliés d'un trait.

Nous avons obtenu à Reims deux pontes, chacune formée d'un œuf double (coque contenant deux ovules) et d'un œuf simple. Les œufs doubles ont un poids relativement élevé par rapport à leur longueur (fig. 9). Ils sont sensiblement plus allongés que leurs jumeaux (fig. 9 et 10), sans être plus larges que des cufs de roussette normaux. Cette règle s'applique aussi aux dimensions de leur chambre chalazifère (fig. 11), nettement plus longue mais de largeur tout à fait normale.

En dehors de ces cas particuliers, les relations statistiques observées sont simples : - une corrélation linéaire entre poids ef longueur (fig. 9), avec des types extrêmes situés environ à $3 \mathrm{~g}$ pour $44 \mathrm{~mm}$, et $5 \mathrm{~g}$ pour $59 \mathrm{~mm}$ de longueur ;

- absence de toute variation de la largeur en fonction de la longueur (la moyenne est de $17 \mathrm{~mm}$ à l'endroit le plus large, $13,5 \mathrm{~mm}$ à l'avant) (fig. 10) ;

- faible corrélation positive entre longueur de la chambre chalazifère et longueur de la coque (fig. 11) ; comme la largeur de la coque, celle de la chambre est également indépendante de la longueur.

Pour ce qui concerne la surface de la coque (fig. 12), elle varie de 12 à $18 \mathrm{~cm}^{2}$. Le poids de la coque vide (sans les filaments) oscille entre 0,35 et $0,75 \mathrm{~g}$, soit en gros le $1 / 10^{e}$ du poids de l'œuf qui est essentiellement déterminé par la masse de son contenu. Si l'on admet que l'origine théorique de la courbe passe par l'origine des axes des coordonnées, la relation entre poids et surface de la coque vide doit être une relation d'allométrie positive.

La disposition des mesures autour de cette courbe (ou d'une droite d'ajustement, si l'on préfère) est attribuée aux variations d'épaisseur, déjà mentionnées (§2). On notera toutefois que les données de ce graphique concernent uniquement des œufs obtenus à Banyuls.

Des échantillons de 1 à $2 \mathrm{~cm}^{2}$ ont été découpés dans les faces de ces coques. Représentée sur une échelle dilatée (facteur multiplicatif identique pour chaque axe), la relation poids-surface de ces échantillons se trouve sensiblement alignée sur la relation valable pour les coques entières (fig. 12), ce qui montre que les épaississements 


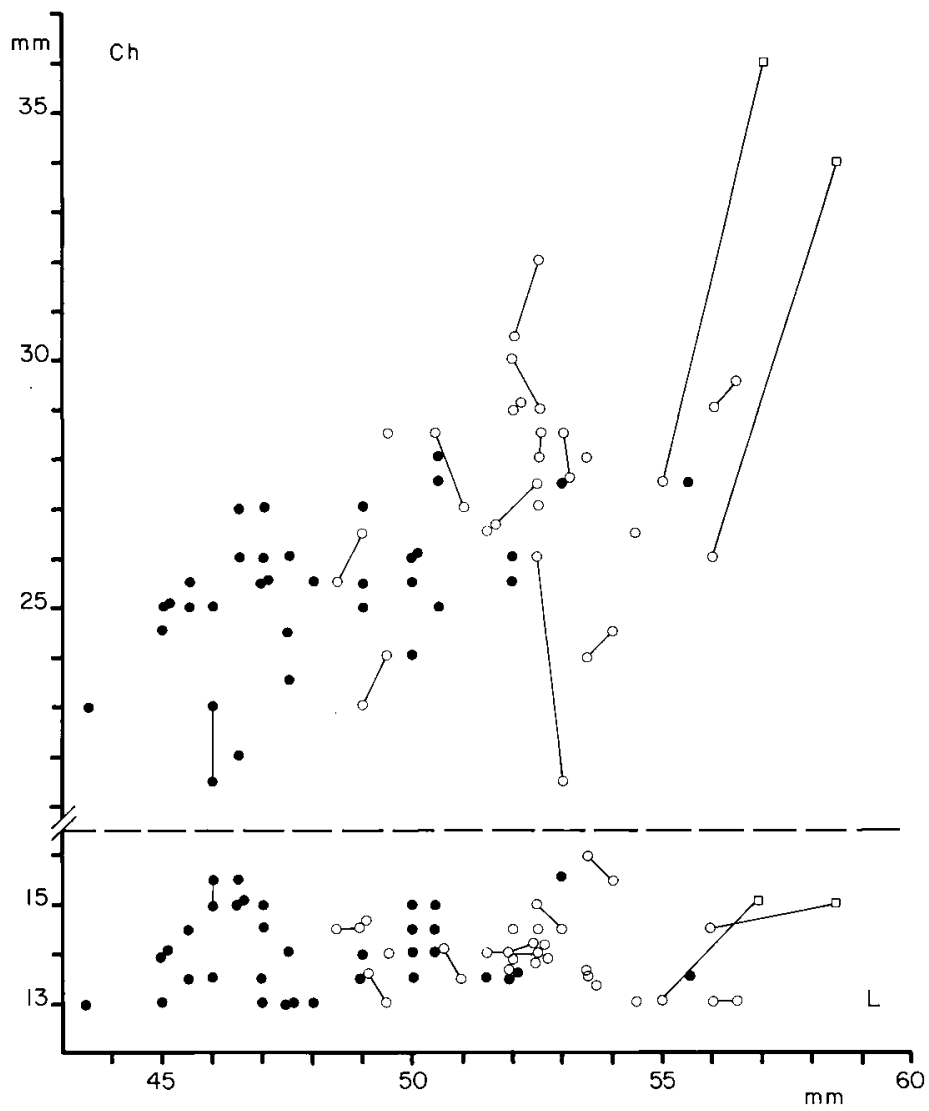

FIG. 11. - Relation entre la longueur de la chambre chalazifère ( $\mathrm{Ch}$, en $\mathrm{mm}$ ), ou sa largeur (en bas, sous les tirets), et la longueur de l'œuf ( $(\mathrm{L}, \mathrm{mm})$. Symboles comme dans la figure 7 . Relation du haut : très significative $(r=0,55)$.

de la coque (arêtes) n'apportent qu'un faible supplément de poids. L'épaisseur de la coque présente de nombreuses irrégularités autant que des variations individuelles

\section{Discussion et conclusions.}

L'œuf de roussette a des dimensions assez variables. Cette variabilité porte essentiellement sur la longueur de la coque et de la chambre chalazifère. Il est remarquable que la largeur, au contraire reste fixe, ce qui impose aux ovules leur forme allongée dans l'œuf.

Le cas des œufs doubles, véritable "expérience naturelle», montre clairement qu'il existe un mécanisme local d'ajustement de la longueur de la coque et de la chambre chalazifère à la longueur des deux ovules accolés parvenus en même temps dans une glande nidamentaire. La coque normale, élaborée dans la glande symétrique, nous sert de témoin. 


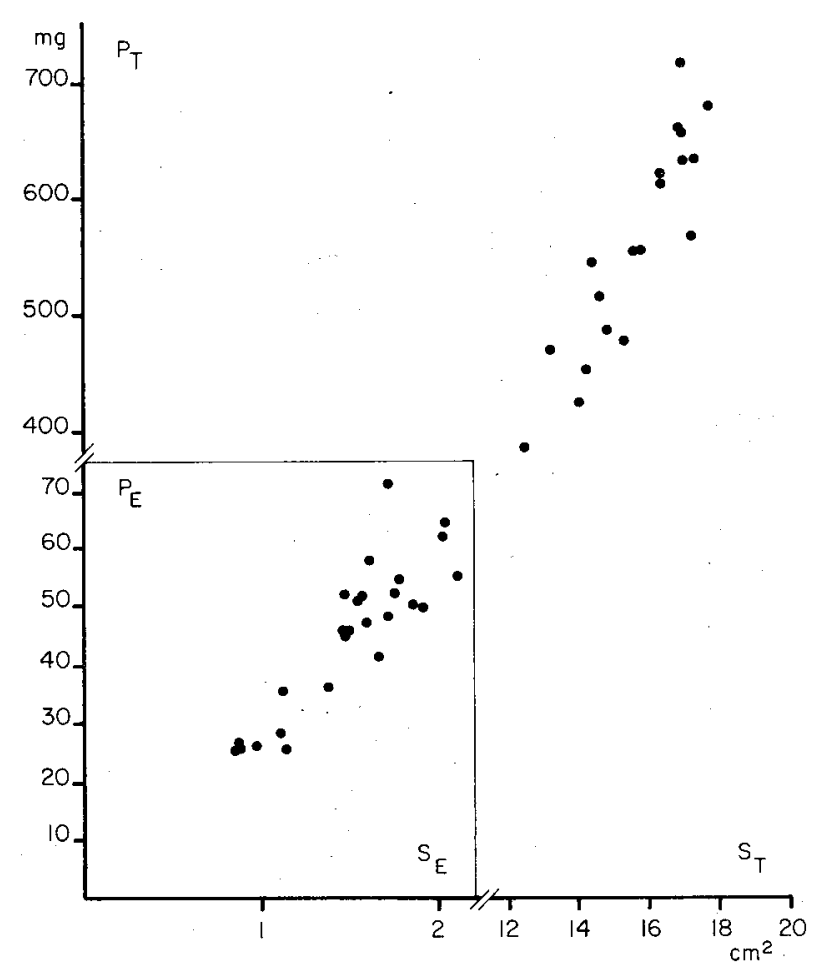

FIG. 12. - Reiation poids ( $\left.\mathrm{P}_{\mathrm{T}}\right)$-surface $\left(\mathrm{S}_{\mathrm{T}}\right)$ de la coque entière, ou $\left(\mathrm{P}_{\mathrm{E}}, \mathrm{S}_{\mathrm{E}}\right)$ d'un échantillon de $1-2 \mathrm{~cm}^{2}$ découpé dans sa face latérale. L'échelle est multipliée par le facteur 5 pour la relation $P_{E}=f\left(S_{E}\right)$. Les œufs proviennent tous de Banyuls.

La fixité de la largeur des coques, contrastant avec la grande variabilité de leur longueur, existe bien dans la population naturelle comme le montrent les œufs recueillis à Banyuls, mais elle se trouve soulignée dans notre lot par l'addition des œufs de grande taille venant de l'élevage. Cette fixité correspond à une variation de $3 \mathrm{~mm}$ seulement pour les deux largeurs mesurées, tandis que les écarts atteignent une amplitude de $16 \mathrm{~mm}$ pour la longueur des œufs (fig. 10). Les raisons anatomiques de cette limitation dans la largeur des coques doivent être recherchées dans la structure des glandes nidamentaires, dont le calibre serait déterminé assez strictement. Dans ces conditions, une intensité plus grande de la sécrétion ne pourrait se traduire que par l'allongement des coques. Cette intensité peut avoir un rapport avec l'épaisseur de la paroi des glandes, dont on connaît les variations physiologiques considérables (Mellinger, 1966).

Le petit nombre de pondeuses réunies dans un seul bac en élevage nous a permis de noter la présence de séries d'œufs d'aspect semblable (forme, pigmentation, transparence). Nous avions donc supposé que chaque femelle produisait un type de coque déterminé durant des mois, sinon des années. Mais les mensurations réalisées en 19781979 (fig. 9 et suivantes) ne permettent pas de reconnaître des classes bien distinctes. 
Disposant d'un nouveau lot de quatre pondeuses au printemps de 1980, nous observons actuellement quatre types d'œufs bien différents. L'une des femelles ne pond que des œufs de 53,5-57 × 19,5-21 mm (largeur très supérieure aux données de la fig. 10). Les autres sont de dimensions normales $(48,5-53 \times 17-18,5 \mathrm{~mm}$, la plupart autour de $52 \times 17,5 \mathrm{~mm}$ ). De plus, la mensuration des « jaunes» indique que cette différence de taille des coques n'est pas étroitement liée à une différence dans le volume des ovules.

Ceci montre donc l'influence du choix des pondeuses dans les fluctuations observées en élevage. Le rôle des facteurs d'environnement (eau, nourriłure, température, lumière) n'apparaît pas clairement. Si l'étude das œufs doubles met bien en évidence un phénomène d'ajustement des dimensions de la coque en fonction de son contenu, le volume des ovules logés dans les œufs simples est assez consłant pour qu'il apparaisse comme un facteur secondaire dans la variabilité biométrique observée. Son influence mériterait toutefois d'être précisée par une étude plus approfondie des variations de la masse des ovules dans des coques de longueurs extrêmes.

Les différences d'épaisseur de la paroi des coques, découvertes récemment, n'ont pas encore fait l'objet d'une étude précise. Par contre, les courbes de croissance embryonnaires (fig. 6) ont été comparées avec celles obtenues en 1971 (AlluchonGérard et Mellinger). La figure 13 montre que la taille est nettement supérieure (de 3 à $4 \mathrm{~mm}$ ) avant le 150e jour, en eau de mer artificielle. La réalité d'un éventuel effet de celle-ci reste à établir expérimentalement. En toute hypothèse, il ne s'agit que d'une différence faible.

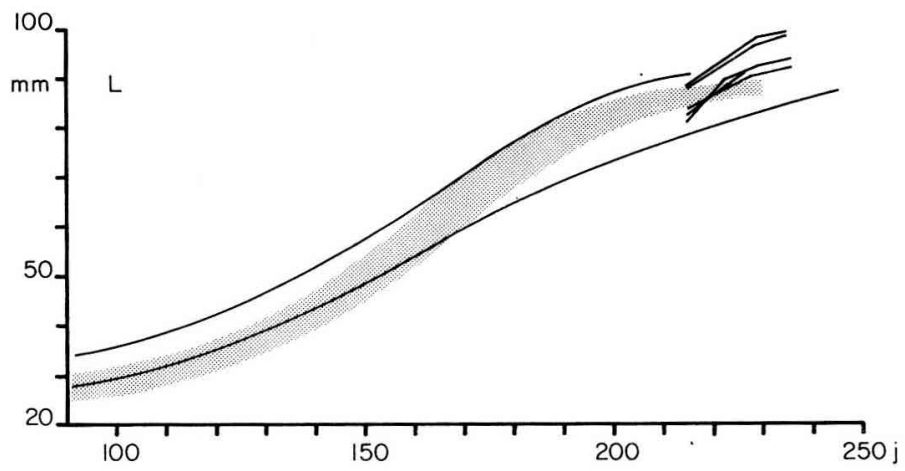

FIG. 13. - Différences dans la distribution des courbes de croissance embryonnaires établies en 1971 (en eau de mer nafurelle, cf. Alluchon-Gérard et Mellinger, 1971 ; zone tramée) ef présentement (courbes de la fig. 6, sauf la plus haute, déviante ; autres courbes non figurées ; lignes courbes confinues = limites de distribution). On a ajouté (en haut à droite, tracés anguleux) cinq courbes de croissance partielles montrant la reprise de la croissance après l'éclosion naturelle, reprise également perceptible chez certains embryons élevés hors de leur coque (fig. 6).

Comme Krishnan (1959) l'a déjà figuré pour un autre Scyliorhinidae, Chiloscyllium griseum, il existe trois couches bien distinctes dans la paroi de la coque chez Scyliorhinus canicula, et nous avons également vérifié leur présence dans l'espèce voisine Scyliorhinus stellaris. Knight et Hunt (1976) n'ont pas signalé la mince couche interne. Celle-ci a été découverte chez la roussette par Rusaouën (1976), qui la nomme L4. Cet auteur, comme l'avait fait Fauré-Fremiet (1938), établit des subdivisions parmi 
les lames formant la couche moyenne. Elle distingue, en particulier, une couche L3, que nous n'avons jamais pu mettre en évidence sur des coques fraîches (non fixées). Une connaissance précise de la structure de cette coque est indispensable pour pouvoir analyser les modalités de sa sécrétion.

Reçu en mars 1980.

Accepté en mai 1980.

\section{Références}

ALLUCHON-GÉRARD M. J., MELLINGER J., 1971. Mise en évidence de plusieurs phases distinctes dans la croissance embryonnaire de la roussette (Scyllium canicula Cuv.). Ann. Embryol. Morphog., 4, 19-35.

BOTTE V., BASILE C., GRANATA G., 1976. Osservazioni sull'attività « albuminipara » della ghiandola nidamentale del Selacio oviparo, Scylliorhinus stellaris. Publ. Staz. Zool. Napoli, 40, 255-259.

FAURÉ-FREMIET E., 1938. Structure de la capsule ovulaire chez quelques Sélaciens. Arch. Anat. micr., 34, 23-51.

FOULLEY M. M., MELLINGER J., 1980. La diffusion de l'eau tritiée, de l'urée-14C el d'autres substances à travers la coque de l'œuf de roussette, Scyliorhinus canicula. C. R. Acad. Sci., Paris, sér. D, 290, 427-430.

JOSEPH H., 1906. Ein Doppelei von Scyllium nebst Bemerkungen über die Entwicklung. Anat. Anz., 29, 367-372.

KNIGHT D. P., HUNT S., 1976. Fine structure of the dogfish egg case : a unique collagenous material. Tissue Cell, 8, 183-193.

KRISHNAN G., 1959. Histochemical studies on the nature and formation of egg capsules of the shark Chiloscyllium griseum. Biol. Bull., 117, 298-307.

LELOUP J., OLIVEREAU M., 1951. Données biométriques comparatives sur la roussette (Scyllium canicula L.) de la Manche et de la Méditerranée. Vie et Milieu, 2, 182-209.

MELLINGER J., 1966. Etude biométrique et histophysiologique des relations entre les gonades, le foie et la thyroïde chez Scyliorhinus coniculus (L.). Cahier Biol. mar., Roscoff, 7, 107-137.

MELLINGER J., FOULLEY M. M., 1979. L'utilisation de l'œuf de petite roussette, Scyliorhinus canicula (L.), en embryologie expérimentale et en physiologie embryonnaire. Ann. Soc. fr. Biol. Dév. Suppl. «Expérimentation sur les organismes marins élevés en laboratoire », 61-70.

OUANG TE YIO, 1931. La glande de l'éclosion chez les plagiosiomes. Ann. Inst. océanogr. Monaco, 10, $281-370$.

RUSAOUËN M., 1976. The dogfish shell gland, a histochemical study. J. exp. mar. Biol. Ecol., 23, 267-283.

RUSAOUËN M., 1978. Etude ultrastructurale des zones à sécrétions protéiques et glycoprotéiques de la glande nidamentaire de la roussette à maturité. Arch. Anat. micr. Morph. exp., 67, 107-119.

RUSAOUËN M., PUJOL J. P., BOCQUET J., VEILLARD A., BOREL J. P., 1976. Evidence of collagen in the egg capsule of the dogfish, Scyliorhinus canicula. Comp. Biochem. Physiol., 53 B, 539-543.

VAYSSIÈRE A., 1909. Note sur un œuf double de squale. C. R. Soc. Biol. Paris, 67, 872-873.

WINTREBERT P., 1920a. L'embryon de Scylliorhinus canicula L. Gill considéré comme animal de laboratoire. Bull. Soc. Zool. France, 45, 311-341.

WINTREBERT P., 1920b. La contraction rythmée aneurale des myotomes chez les embryons de Sélaciens. I. Observation de Scylliorhinus conicula L. Gill. Arch. Zool. exp. génér., 60, 221-460.

WINTREBERT P., 1927o. Sur la présence d'une membrane chalazifère dans l'œuf des Sélaciens squaliformes. C. R. Soc. Biol. Poris, 96, 252-255.

WINTREBERT P., 1927b. L'ascension du germe au pôle dorsal du vitellus pendant le développement, chez les Plagiostomes (Scylliorhinus canicula L. Gill). C. R. Soc. Biol. Paris, 96, 668-671.

WINTREBERT P., 1927c. Les membranes vitellines et chalazifères au cours du développement dans l'œuf des Sélaciens. C. R. Soc. Biol. Paris, 96, 852-854.

WINTREBERT P., 1931. La dissolution des glaires ef du gluten dans l'œuf des Plagiostomes et la recherche des facteurs qui la déterminent. C. R. Soc. Biol. Paris, 107, 1447-1450.

WOURMS J. P., 1977. Reproduction and development in Chondrichthyan fishes. Amer. Zool., 17, $379-410$. 